Supporting Information

\title{
Deterministic sorting of submicron particles and extracellular vesicles using a combined electric and acoustic field
}

\author{
Mahnoush Tayebi ${ }^{1}$, Dahou Yang ${ }^{1}$, David J. Collins ${ }^{2 *}$ and Ye Ai ${ }^{*}$
}

${ }^{1}$ Pillar of Engineering Product Development, Singapore University of Technology and Design, Singapore 487372, Singapore

${ }^{2}$ Department of Biomedical Engineering, The University of Melbourne, Melbourne, VIC 3010, Australia

Corresponding authors*

Email: aiye@sutd.edu.sg, Tel: (+65) 64994553

Email: david.collins@unimelb.edu.au, Tel: (+61) 383440867 


\section{Methods}

\subsection{Experimental set up}

The devices used in this work are comprised of a $2 \mathrm{~mm}$-wide-microfluidic chamber integrated with a $128^{\circ}$ Y-cut, X-propagation piezoelectric lithium niobate $\left(\mathrm{LiNbO}_{3}\right)$ substrate on which a series of gold interdigital transducer (IDT) electrodes were deposited via electron-beam evaporation, consisting of an adhesion layer (10 $\mathrm{nm}$ thick chromium) followed by the conductive layer (100 nm thick gold). To enhance the microchannel/IDT chip bond strength a thin layer of $\mathrm{SiO}_{2}(150 \mathrm{~nm})$ was subsequently deposited on all chip areas. After fabrication of the gold electrodes on the piezoelectric substrate, the whole wafer was placed back in the electron-beam evaporator for $\mathrm{SiO}_{2}$ deposition with $150 \mathrm{~nm}$ thickness. The thin layer of $\mathrm{SiO}_{2}$ is orders of magnitude smaller than the applied wavelength and has little or no effect on wavefront attenuation ${ }^{1}$. Such a layer is widely used to prevent electrode corrosion, biofouling and electrical degradation via an electrolytic media without compromising the DEP field ${ }^{2}$, 3. The number of finger pairs for a $120 \mu \mathrm{m}$ and $80 \mu \mathrm{m}$ SAW wavelength was calibrated at 30 and 24 finger pairs to match the electrical impedance with a $50 \Omega$ signal generator and the applied frequency was $32.4 \mathrm{MHz}$ and $48.5 \mathrm{MHz}$, respectively. The microfluidic chamber was prepared via photolithography method using an SU-8 on silicon mold followed by polydimethylsiloxane (SYLGARD 184, Aldrich) soft lithography to make the microchannel. The 29 and $43 \mu \mathrm{m}$ height polydimethylsiloxane (PDMS) microfluidic chambers were bonded to the lithium niobate device after exposing to an air plasma (Harrick Plasma PDC-32G; 1000 mTorr, $18 \mathrm{~W}$ ) for 2 minutes. Two buffer inlets were designed in the channel to confine the main flow in the acoustic/DEP force field and keep the initial position of the inlet flow constant. The microfluidic channels were washed with deionized water containing $1 \%$ F127 surfactant for 10 minutes before each experiment. A syringe pump (KD Scientific, Holliston, MA) was used to introduce the fluid with different flow rates to the microchannel. Sinusoidal electrical signals with different applied powers $(0.02,0.03,0.05,0.08,0.1,0.2,0.3,0.5$ Watt $)$ were generated using a high-frequency signal generator (Rigol DSG815, Beijing, China) and a broadband amplifier (Ophir RF, 0.15-230 MHz 25W RF Broadband Power Amplifier). 


\subsection{Sample preparation}

Fluorescent polystyrene particles with $10 \mu \mathrm{m}, 1 \mu \mathrm{m}, 500 \mathrm{~nm}, 300 \mathrm{~nm}$, and $100 \mathrm{~nm}$ diameters were purchased from Magsphere, USA and suspended in 1\% Pluronic F127 (Sigma-Aldrich, USA) in ultrapure water (Thermofisher, Millipore filtration system) with conductivity of $0.0042 \mathrm{~S} / \mathrm{m}$ or in $1 \times$ phosphate-buffered saline (PBS) with conductivity of $\sim 1 \mathrm{~S} / \mathrm{m}$. Extracellular vesicles were isolated from MCF-7 breast cancer cells which were sub-cultured in Dulbecco's modification of Eagle's medium (DMEM) cell culture medium (supplemented with $10 \%$ fetal bovine serum) for $72 \mathrm{~h}$ in a humidified incubator under $37^{\circ} \mathrm{C}, 5 \%(\mathrm{v} / \mathrm{v}) \mathrm{CO}_{2}$. The supernatant of the cell culture medium was collected from the $90 \%$ confluent cell culture flask and centrifuged at $2000 \mathrm{~g}$ for 30 minutes to remove cell debris before isolating exosomes with Total Exosome Isolation Reagent (Thermofisher scientific) following the manufacture's protocol for the control sample. For vDLD experiments the collected cell culture medium was introduced directly to the microchannel without pre-filtration/centrifugation.

\subsection{Scanning electron microscopy}

To prepare the sample for dehydration step, the purified exosomes by vLD device were mixed with a precipitation reagent (Thermofisher scientific) with ratio of $1: 1.5$ and were centrifuged at $10000 \mathrm{~g}$ for $1 \mathrm{~h}$ after overnight incubation in $4{ }^{\circ} \mathrm{C}$. The pellet was transferred to the SEM stab and were dehydrated in a freeze drier before conducting SEM imaging.

\subsection{Nanoparticle tracking analysis}

Nanoparticle tracking analysis (NTA) of the collected samples were conducted by using ZetaView, Particle Metrix GmbH system. A calibration procedure according to the manufacture's protocol was conducted before all experiments. The setting parameters were adjusted to include all populations of particles in each experiment. The applied frame rates in NTA videos recording for particles $>300 \mathrm{~nm}$ and $<300 \mathrm{~nm}$ are $30 \mathrm{frame} / \mathrm{s}$ and $7.5 \mathrm{frame} / \mathrm{s}$, respectively.

\section{Theory and working mechanism}


Both ARF and DEP force fields are generated by applying an A/C signal across the IDT electrodes. Implementing the electrodes in the vicinity of the particle suspension permits the simultaneous generation of the electric filed by applying the acoustic field without the need for any additional fabrication steps, alignment or control processes. In this system particles are subjected to three different forces; fluid drag force (along the flow direction), acoustic radiation forces and dielectrophoretic forces (these latter two being perpendicular to the IDT electrode axis). Although this configuration permits generation of both standing and travelling surface acoustic waves (see Supporting Video S1), the travelling wave elements are negligible due to the small size of the particles compare to the wavelength in the fluid. Acoustic manipulation of particles with a Helmholtz number $\left(\mathrm{k}_{\text {Helmholtz }}=\pi \mathrm{d} / \lambda_{f}\right.$, where $\mathrm{d}$ is the particle diameter and $\lambda_{f}$ is the wavelength in the fluid) larger than $\sim 1.4$ results in traveling wave forces dominating particle motion. Conversely, due to the nonlinear scaling of traveling wave forces, standing waves overwhelmingly dominate particle trajectories below this number ${ }^{4-6}$. Table S1 shows the $\mathrm{k}_{\mathrm{Helmholtz}}$ factor for particle diameters used in this work, with $\mathrm{k}_{\mathrm{Helmholt}}<1$ for all cases, confirming the relative dominance of standing wave acoustic radiation forces in our system. Applying acoustic/DEP force field at an angle to the inlet flow causes particles to deviate towards the pressure nodes/minimum electric fields which gives rise to a drift-mode lateral displacement of the particles, moving them away from the centerline of the channel where maximum local fluid velocities/drag force occur. The acoustic and DEP forces scale with $\mathrm{R}^{3}$, while the drag force scales with $\mathrm{R}$, such that larger particles continue to translate along the flow direction with longer residence times in individual acoustic/DEP force minima, resulting in different lateral positions for different particle sizes (see Figure 1). As particles gradually migrate away from the channel centre, the relative drag force decreases owing to a parabolic nature of the velocity profile. As Wang and Riaud ${ }^{7}$ demonstrated, the highest precision of sorting occurs at $0^{\circ}$ angle where the electrodes are located orthogonal to the flow but at the cost of the throughput and application of a high power. However, employing a tilted force field at the vicinity of $\sim 45^{\circ}$ gives the opportunity for sorting a wide range of particle sizes with maximum sensitivity through a drift-mode deviation of the particles. In our vDLD system, employing $50^{\circ}$ tilted angle force fields is combined with a multi-outlet channel design to permit the separation of particles with small 
lateral displacements from the inlet stream. Our channel is further designed to increase pressure drop ( $\Delta P)$ uniformity across different outlets via the use of long and thin channel sections near the outlets, resulting in small differences in flow rate between outlet channels (as $\left.Q_{1} / Q_{2}=\Delta P_{1} / \Delta P_{2}\right)^{8}$. Figure $\mathrm{S} 3$ demonstrates the pressure drops through each outlet using a laminar flow simulation, where the ratio of pressure drops vary from 1 to 1.056 in different outlets, and shows the resulting uniform flow distribution.

The standing wave acoustic field generates a pressure field which gives rise to the acoustic radiation force acting on the particles. To obtain this force, an approximation of a one-dimensional distribution for the pressure field in the microfluidic channel can be considered which gives the acoustic radiation force as

$$
\begin{aligned}
& \mathrm{F}_{\mathrm{aco}}^{x}=-\left(\frac{\pi \mathrm{P}_{0}^{2} \mathrm{~V}_{\mathrm{p}} \beta_{\mathrm{m}}}{2 \lambda}\right) \phi(\beta, \rho) \sin \left(\frac{4 \pi \mathrm{x}}{\lambda}\right), \\
& \phi(\beta, \rho)=\frac{5 \rho_{\mathrm{p}}-2 \rho_{\mathrm{m}}}{2 \rho_{\mathrm{p}}+\rho_{\mathrm{m}}}-\frac{\beta_{\mathrm{p}}}{\beta_{\mathrm{m}}}, \\
& \mathrm{P}_{0}=\sqrt{\alpha \mathrm{P}_{\mathrm{IDT}} \rho_{\mathrm{s}} \mathrm{C}_{\mathrm{s}} / \mathrm{A}}
\end{aligned}
$$

where $V_{p}, \rho_{p}, \beta_{p}$ are particles volume, density and compressibility, $\rho_{m}, \beta_{m}$ are the density and compressibility of the medium, $\mathrm{P}_{0}$ is the acoustic pressure amplitude and $\phi(\beta, \rho)$ represent the acoustic contrast factor. In eqn (S1c), $\mathrm{P}_{\mathrm{IDT}}, \alpha, \rho_{\mathrm{s}}, \mathrm{C}_{\mathrm{s}}$ and $\mathrm{A}$ represent the power of the IDTs, power conversion efficiency, density and the sound speed in the substrate and working area, respectively (see SI Table S2).

Applying a nonuniform electric field on the polarizable particles also gives rise to a DEP force in which its magnitude and direction depends on the size of the particles and the dielectric properties such as frequency-dependent dielectric permittivity of the homogenous spherical particles $\left(\varepsilon_{\mathrm{p}}^{*}\right)$ and medium ( $\left.\varepsilon_{\mathrm{m}}^{*}\right)$,

$$
\left\langle\mathrm{F}_{\mathrm{DEP}}\right\rangle=2 \pi \varepsilon_{0} \varepsilon_{\mathrm{r}, \mathrm{m}} \mathrm{R}_{\mathrm{p}}^{3} \operatorname{Real}[\mathrm{CM}] \nabla\left|\mathrm{E}_{\mathrm{rms}}\right|^{2},
$$




$$
\begin{aligned}
& C M=\frac{\varepsilon_{\mathrm{p}}^{*}-\varepsilon_{\mathrm{m}}^{*}}{\varepsilon_{\mathrm{p}}^{*}+2 \varepsilon_{\mathrm{m}}^{*}} \\
& \varepsilon_{\mathrm{p} \text { or } \mathrm{m}}^{*}=\varepsilon_{0} \varepsilon_{\mathrm{r}, \mathrm{x}}-\mathrm{j}\left(\frac{\sigma_{\mathrm{x}}}{\omega}\right) .
\end{aligned}
$$

The CM here is Clausius-Mossotti factor, which describes the frequency dependence of the DEP force and determines whether force is $\mathrm{nDEP}$ (away from the electrodes) or $\mathrm{pDEP}$ (towards the electodes). $\mathrm{R}_{\mathrm{p}}$ and $\mathrm{E}_{\mathrm{rms}}$ are the radius of the particle and the root mean squared value of the electric field, respectively. In equation (2c), j $=\sqrt{-1}, \varepsilon_{\mathrm{r}, \mathrm{x}}$ and $\sigma_{\mathrm{x}}$ are the relative permittivity and conductivity of the particles $(\mathrm{x}=\mathrm{p})$ or medium $(\mathrm{x}=\mathrm{m})$, respectively, and $\varepsilon_{0}$ is the permittivity of the free space. The particle conductivity here is a function of the medium conductivity and crossover frequency $\left(f_{\text {cof }}\right.$, the frequency at which DEP force reverses its direction), and is given by ${ }^{9}$ :

$$
\begin{aligned}
& \sigma_{p}=\sigma_{s}+\frac{2 K_{s}}{R_{p}}, \\
& K_{s}=\frac{R_{p}}{4}\left(-\sigma_{m}+\sqrt{9 \sigma_{m}^{2}-4\left(\varepsilon_{p}-\varepsilon_{m}\right)\left(\varepsilon_{p}+2 \varepsilon_{m}\right)\left(2 \pi f_{c o f}\right)^{2}}\right),
\end{aligned}
$$

where $\sigma_{s}, \mathrm{~K}_{s}$ and $\mathrm{R}_{p}$ are bulk conductivity, surface conductance and radius of the particles, respectively, and where the value of $\sigma_{s}$ for polystyrene particles is negligible (see SI Table S3). To calculate the surface conductance using equation (3b), it is required to obtain the crossover frequency $\left(f_{c o f}\right)$. We extracted this value from reported experimental values in which $f_{\text {cof }}$ varies linearly (between 50 to 250 $\mathrm{kHz}$ ) by changing the medium conductivity from 0 to $1 \mathrm{~S} / \mathrm{m}^{10}$.

Particles travelling in the fluid with a velocity of $\mathrm{u}_{\mathrm{f}, \mathrm{Y}}$ further experience Stoke's drag that generates a force along the flow direction. Here, $\mathrm{u}_{\mathrm{f}, \mathrm{y}}$ is defined in the $\{\mathrm{x}, \mathrm{y}, \mathrm{z}\}$ coordinate system aligned with the flow direction, where $\mathrm{x}, \mathrm{y}$ and $\mathrm{z}$ are the coordinates in the channel width, length and height directions, respectively. To simulate behavior of the particles under the influence of the DEP and acoustic force fields, $\{\mathrm{X}, \mathrm{y}, \mathrm{Z}\}$ coordinate system is converted to $\left\{\mathrm{X}^{\prime}, \mathrm{Y}^{\prime}, \mathrm{Z}^{\prime}\right\}$ by a rotational transformation with angle $\theta=50^{\circ}$ aligned with the IDT electrodes, where $\mathrm{X}^{\prime}, \mathrm{Y}^{\prime}$ and $\mathrm{Z}^{\prime}$ are the coordinates in the IDTs length, transducer aperture and channel height directions, respectively (see Figure 1). In this new coordinate 
system, the fluid velocity, $\mathrm{u}_{\mathrm{f}, \mathrm{y}}$, can be decomposed into $\mathrm{u}_{\mathrm{f}, X^{\prime}}=\mathrm{V}_{\mathrm{para}} \sin \theta$ and $\mathrm{u}_{\mathrm{f}, Y^{\prime}}=\mathrm{V}_{\mathrm{para}} \cos \theta$, where $\mathrm{V}_{\text {para }}$ is determined by

$$
\mathrm{V}_{\text {para }}=u_{f, y}^{\max }\left(1-\left(\mathrm{X} / \mathrm{x}^{\max }\right)^{2}\right) \text {. }
$$

Behavior of the particles under the influence of drag, DEP and acoustic forces can be characterized by examining the velocity of the particles in the $\left\{\mathrm{X}^{\prime}, \mathrm{Y}^{\prime}, \mathrm{Z}^{\prime}\right\}$ coordinate system. In this coordinate system particle migration entirely in the $Y^{\prime}$ direction is equal to the undisturbed fluid velocity in this direction $\left(\mathrm{u}_{\mathrm{p}, Y^{\prime}}=\mathrm{u}_{\mathrm{f}, Y^{\prime}}\right)$ as the DEP/acoustic forces only act in $\mathrm{X}^{\prime}$ direction which dictate velocity of the particles in $\mathrm{X}^{\prime}$ coordinate. This velocity can be obtained based on the balance between the forces on a particle $\vec{F}_{\text {drag }}=\vec{F}_{\text {aco }}+\vec{F}_{\text {DEP. }}$ Equation S5 represents the drag force which pushes particles with radius $R_{p}$ forward along the fluid flow direction with viscosity of $\mu$

$$
\mathrm{F}_{\mathrm{drag}}=-6 \pi \mu \mathrm{R}_{\mathrm{p}}\left(\mathrm{u}_{\mathrm{p}, X^{\prime}}-\mathrm{u}_{\mathrm{f}, X^{\prime}}\right) \text {, }
$$

where $\mathrm{u}_{\mathrm{p}, X^{\prime}}$, is the particle velocity in $\mathrm{X}^{\prime}$ direction. Including all forces results in this velocity being expressed as

$$
\begin{aligned}
& u_{\mathrm{p}, X^{\prime}}=\mathrm{u}_{\mathrm{f}, X^{\prime}}+\mathrm{R}_{\mathrm{p}}^{2} / 3 \mu\left(\frac{\mathrm{P}^{2} \pi \beta_{\mathrm{f}} \phi(\beta, \rho) \sin (2 \mathrm{kx})}{3 \lambda}+\varepsilon_{\mathrm{m}}[\mathrm{CM}] \nabla\left|\mathrm{E}_{\mathrm{rms}, \mathrm{h} / 2}\right|^{2}\right), \\
& \mathrm{u}_{\mathrm{p}, Y^{\prime}}=\mathrm{u}_{\mathrm{f}, Y^{\prime}} .
\end{aligned}
$$

We calculate the DEP force at $\mathrm{z}_{0}=\mathrm{h} / 2$, where we assume the particles to travel at the center plane of the channel, since the choice of a channel heights used of approximately one fluid wavelength creates a $z$-direction nodal position at this height. To evaluate the particle trajectories, we applied the MATLAB streamline function using the particles velocity field components in equations S6a and b. 
Table S1. The evaluation of $k_{H e l m h o l t z}$ factor

\begin{tabular}{c|ccccc}
\hline Particle size $(\mu \mathrm{m})$ & 0.1 & 0.3 & 0.5 & 1 & 10 \\
\hline Frequency $(\mathrm{MHz})$ & 48.5 & 48.5 & 48.5 & 48.5 & 32.4 \\
\hline $\mathrm{k}_{\text {Helmholtz }}$ & 0.0102 & 0.0305 & 0.0509 & 0.1018 & 0.6799
\end{tabular}


Table S2. Parameters used for the calculation of acoustic radiation force

\begin{tabular}{|c|c|c|c|}
\hline \multicolumn{2}{|c|}{ Viscosity of the medium $(\mathrm{Pa} s)$} & \multirow{2}{*}{$\mu$} & \multirow{2}{*}{$\begin{array}{c}0.001 \\
2.49 \times 10^{-10}\end{array}$} \\
\hline \multirow{4}{*}{ Compressibility $\left(\mathrm{Pa}^{-1}\right)$} & Polystyrene particles & & \\
\hline & Extracellular vesicles & & $3.50 \times 10^{-10}$ \\
\hline & 11 & & \\
\hline & Medium & $\beta_{m}$ & $4.48 \times 10^{-10}$ \\
\hline \multirow{5}{*}{ density $\left(\mathrm{kg} \mathrm{m}^{-3}\right)$} & Polystyrene particles & & 1050 \\
\hline & Extracellular vesicles & $\rho_{p}$ & 1130 \\
\hline & 11 & & \\
\hline & Medium & $\rho_{m}$ & 997 \\
\hline & Substrate & $\rho_{s}$ & 4650 \\
\hline \multicolumn{2}{|c|}{ Sound speed in medium $\left(\mathrm{m} \mathrm{s}^{-1}\right)$} & $c_{m}$ & 1495 \\
\hline \multicolumn{2}{|c|}{ Sound speed in substrate $\left(\mathrm{ms}^{-1}\right)$} & $c_{s}$ & 3931 \\
\hline \multicolumn{2}{|l|}{ IDTs working area $\left(\mathrm{mm}^{2}\right)$} & A & 3.8 \\
\hline \multicolumn{2}{|l|}{ power conversion efficiency } & $\alpha$ & $0.4-0.6$ \\
\hline \multicolumn{2}{|c|}{ Acoustic contrast factor of polystyrene particles } & $\phi_{p}(\beta, \rho)$ & 0.4955 \\
\hline \multicolumn{2}{|c|}{ Acoustic contrast factor of EVs ${ }^{12}$} & $\phi_{e x o}(\beta, \rho)$ & 0.3616 \\
\hline \multicolumn{2}{|l|}{ *Vibration Amplitude } & $\mathrm{u}_{3}$ & $2.63 \mathrm{~nm}$ \\
\hline \multicolumn{4}{|c|}{$\begin{array}{l}* u_{3}=\sqrt{\frac{\Lambda N_{S A W}}{W F_{N}}}, \text { where } \Lambda, \mathrm{N}_{\mathrm{SAW}}, \mathrm{W} \text { and } \mathrm{F}_{\mathrm{N}} \text { are wavelength, power of a plane Rayleigh wave, width of } \\
\text { a plane Rayleigh wave and a piezoelectric substrate specific factor, respectively }{ }^{13} \text {. }\end{array}$} \\
\hline
\end{tabular}


Table S3. Parameters used for the DEP force calculation

\begin{tabular}{|c|c|c|c|}
\hline Permittivity of the fre & & $\overline{\varepsilon_{0}}$ & $8.85 \times 10^{-12}$ \\
\hline \multirow[t]{2}{*}{ Relative permittivity } & $\begin{array}{l}\text { polystyrene } \\
\text { particles }\end{array}$ & $\varepsilon_{r, p}$ & 2.55 \\
\hline & medium & $\varepsilon_{r, m}$ & 78.36 \\
\hline \multirow[t]{2}{*}{ Conductivity } & $\begin{array}{l}\text { *polystyrene } \\
\text { particles }\end{array}$ & $\sigma_{p}$ & $7.7 \times 10^{-3}$ \\
\hline & medium & $\sigma_{m}$ & $0.0042 \mathrm{~S} / \mathrm{m}$ \\
\hline \multicolumn{4}{|c|}{$\begin{array}{l}\text { Frequency-dependent dielectric permittivity for extracellular } \\
\text { vesicles is obtained by using cell's electrical properties in a } \\
\text { single-shell model }{ }^{14} \text {. }\end{array}$} \\
\hline \multicolumn{3}{|c|}{ EVs CM factor (in cell culture medium $1.6 \mathrm{~S} / \mathrm{m}$ ) } & -0.0460 \\
\hline \multicolumn{4}{|c|}{$\begin{array}{l}* \sigma_{p}=\sigma_{b}+\frac{2 k_{s}}{R_{p}} \text {, where the bulk conductivity }\left(\sigma_{b}\right) \text { is a negligible value for the } \\
\text { and } k_{s} \text { represents the surface conductance: } \\
k_{s}=\frac{R_{p}}{4}\left(-\sigma_{m}+\left[9 \sigma_{m}^{2}-4\left(\left(\varepsilon_{0} \varepsilon_{r, p}-\varepsilon_{0} \varepsilon_{r, m}\right)\left(\varepsilon_{0} \varepsilon_{r, p}+2 \varepsilon_{0} \varepsilon_{r, m}\right)(2 \pi f)^{2}\right)\right]^{1 / 2}\right)\end{array}$} \\
\hline
\end{tabular}



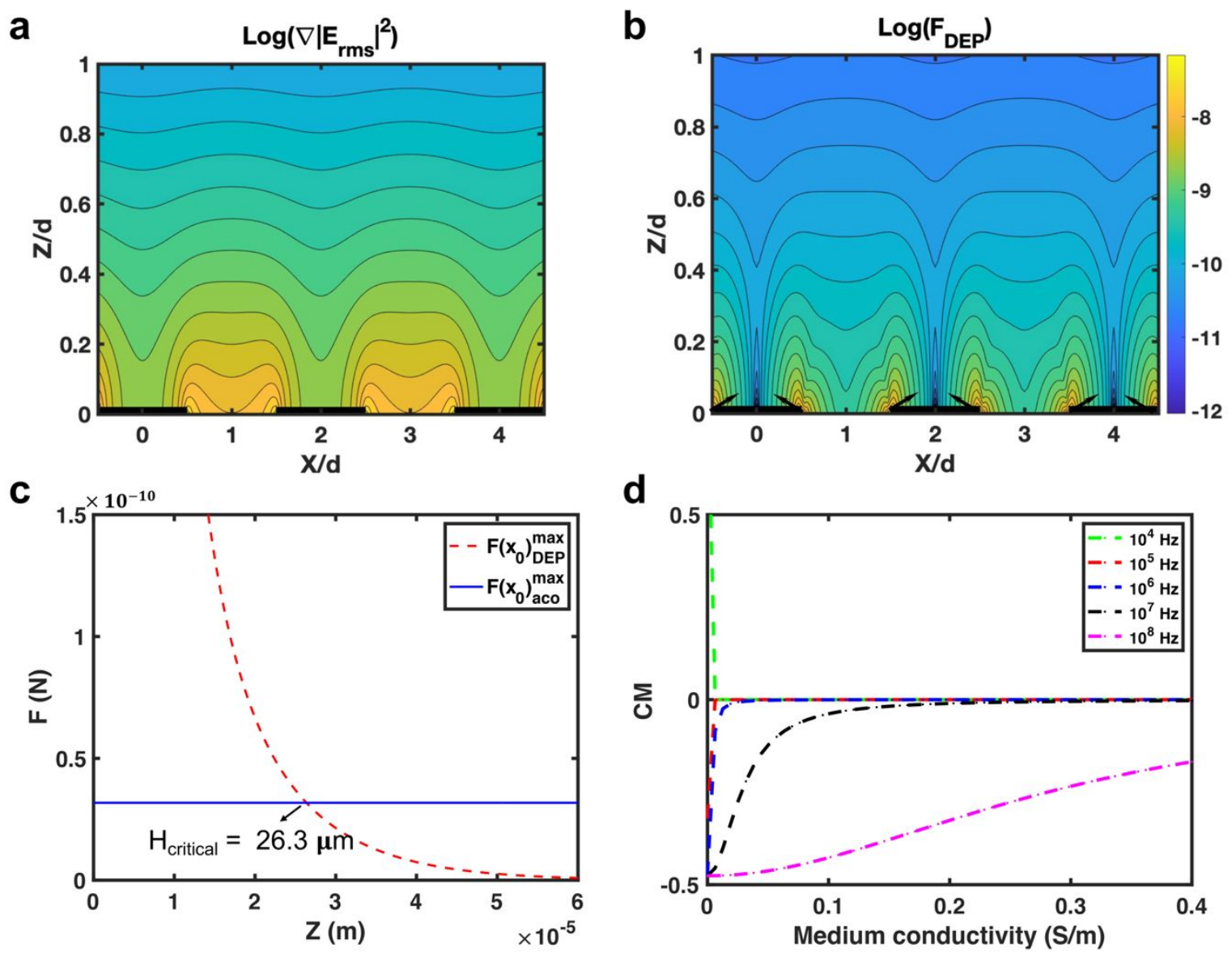

Figure S1. Demonstration of the dielectrophoresis (DEP) fields in our vDLD system. The contour plots of (a) electric field and (b) DEP forces above the electrodes. (c) The critical height of the channel in which the maximum acoustic force is equal to the DEP force. (d) The effect of medium conductivity on the Clausius-Mossotti factor (CM) at different applied frequencies. 


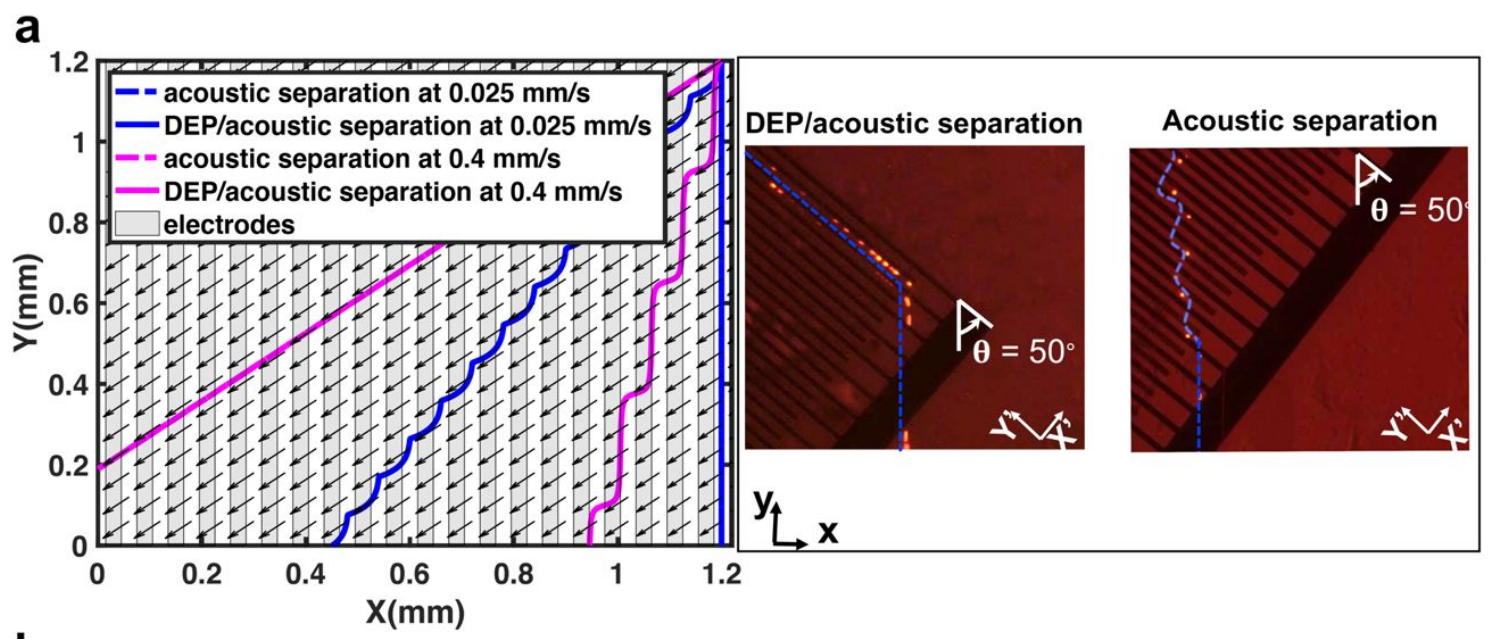

b

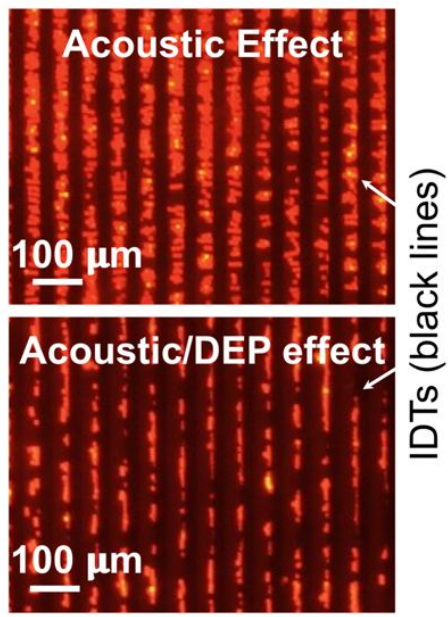

C

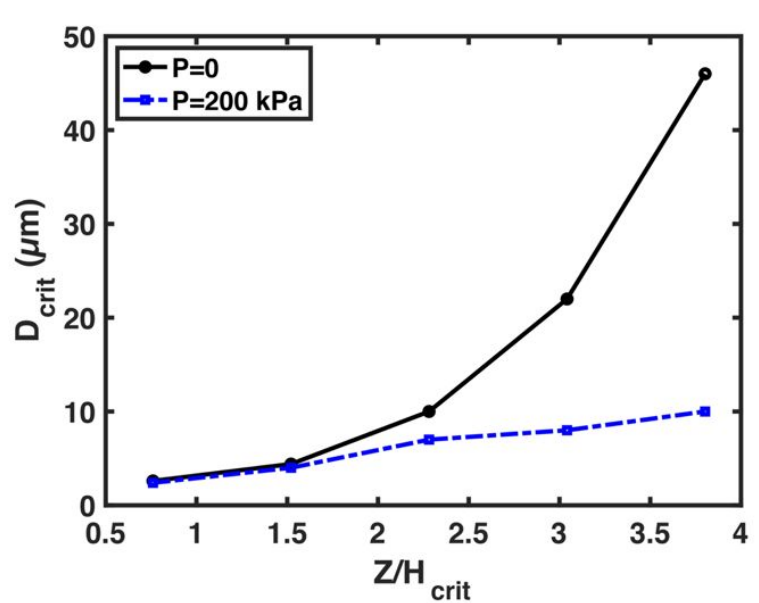

Figure S2. The effect of co-existing DEP and acoustic forces on particles deflection. (a) Trajectory of $10 \mu \mathrm{m}$ particles in a acoustic-based and DEP/Acoustic-based deflection at two different velocities $(\lambda=$ $120 \mu \mathrm{m}$, frequency $=32.4 \mathrm{MHz}$ ). The images at right panel demonstrate the $10 \mu \mathrm{m}$ particles path in two different medium conductivities which employing a low conductivity medium $(0.004 \mathrm{~S} / \mathrm{m})$ results in a larger lateral displacement due to the coexistence of DEP and acoustic forces while applying $1 \times \mathrm{PBS}$ as the medium (conductivity=1 S/m) deflects particles gradually with smaller lateral displacement. (b) Demonstration of the acoustic and combination of the acoustic/DEP effects in static flow condition (c) The effect of combined DEP/acoustic forces on the critical diameter at different vertical position of the particles relative to their critical height. The zero pressure indicates eliminating the acoustic effect while by applying SAW with $200 \mathrm{kPa}$ pressure field, a combined DEP/Acoustic force fields result in a lower critical diameter. 


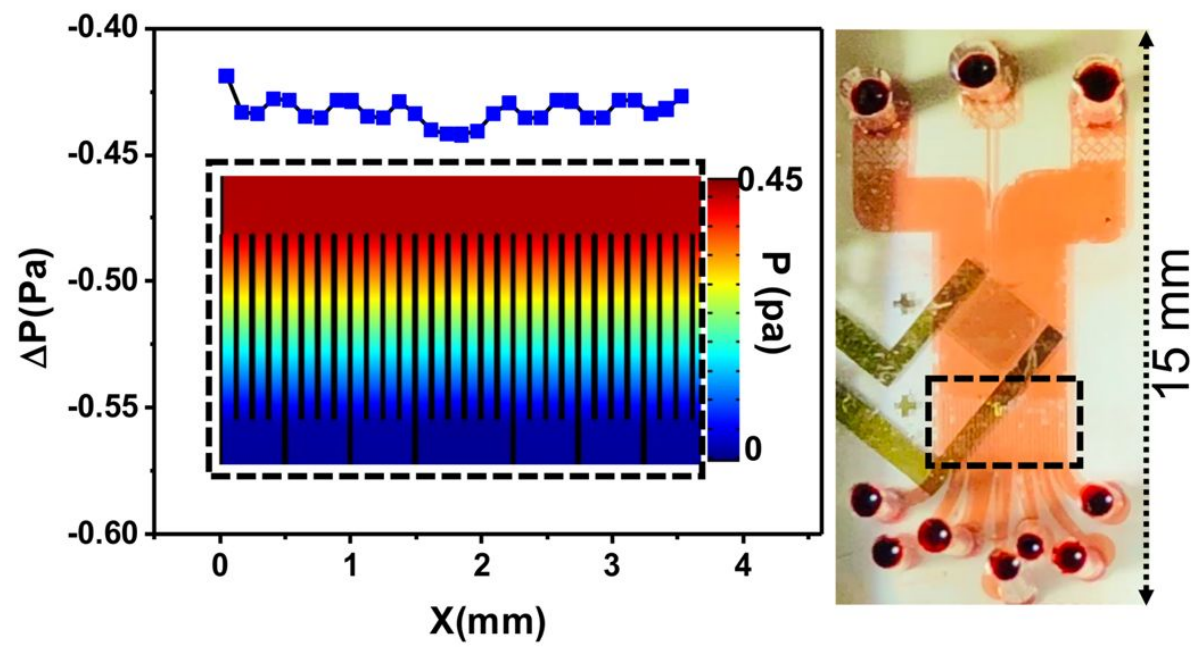

Figure S3. Evaluation of the fluidic pressure drop in the vicinity of channel outlets to assess the variation of the volumetric flow rate across the channel width. 


\section{References}

1. Winkler, A.; Brünig, R.; Faust, C.; Weser, R.; Schmidt, H., Towards efficient surface acoustic wave (SAW)-based microfluidic actuators. Sensors and Actuators A: Physical 2016, 247, 259-268.

2. $\quad$ Yang, L.; Banada, P. P.; Chatni, M. R.; Seop Lim, K.; Bhunia, A. K.; Ladisch, M.; Bashir, R., A multifunctional micro-fluidic system for dielectrophoretic concentration coupled with immunocapture of low numbers of Listeria monocytogenes. Lab on a Chip 2006, 6 (7), 896-905.

3. Emaminejad, S.; Javanmard, M.; Gupta, C.; Dutton, R.; Davis, R.; Howe, R. In Ultra dielectrophoresis: Electrothermal analysis and its applications in microfluidic sample preparation and proteomics, 17th International Conference on Miniaturized Systems for Chemistry and Life Sciences, MicroTAS 2013, Chemical and Biological Microsystems Society: 2013; pp 850-852.

4. Destgeer, G.; Ha, B. H.; Park, J.; Jung, J. H.; Alazzam, A.; Sung, H. J., Microchannel Anechoic Corner for Size-Selective Separation and Medium Exchange via Traveling Surface Acoustic Waves. Analytical Chemistry 2015, 87 (9), 4627-4632.

5. Ma, Z.; Collins, D. J.; Ai, Y., Detachable Acoustofluidic System for Particle Separation via a Traveling Surface Acoustic Wave. Analytical Chemistry 2016, 88 (10), 5316-5323.

6. Collins, D. J.; Devendran, C.; Ma, Z.; Ng, J. W.; Neild, A.; Ai, Y., Acoustic tweezers via sub-time-of-flight regime surface acoustic waves. Science Advances 2016, 2 (7), el600089.

7. Wang, W.; Zhou, J.; Riaud, A., Trajectory of particles exposed to a tilted-angle washboard potential: application to high-throughput acoustophoresis in microchannels. arXiv preprint arXiv:1902.08892 2019.

8. Tanyeri, M.; Ranka, M.; Sittipolkul, N.; Schroeder, C. M., A microfluidic-based hydrodynamic trap: design and implementation. Lab on a Chip 2011, 11 (10), 1786-1794.

9. Chen, Q.; Yuan, Y. J., A review of polystyrene bead manipulation by dielectrophoresis. RSC Advances 2019, 9 (9), 4963-4981.

10. Arnold, W.; Schwan, H.; Zimmermann, U., Surface conductance and other properties of latex particles measured by electrorotation. Journal of Physical Chemistry 1987, 91 (19), 5093-5098.

11. Lee, K.; Shao, H.; Weissleder, R.; Lee, H., Acoustic Purification of Extracellular Microvesicles. ACS Nano 2015, 9 (3), 2321-2327.

12. Wu, M.; Ouyang, Y.; Wang, Z.; Zhang, R.; Huang, P.-H.; Chen, C.; Li, H.; Li, P.; Quinn, D.; Dao, M.; Suresh, S.; Sadovsky, Y.; Huang, T. J., Isolation of exosomes from whole blood by integrating acoustics and microfluidics. Proceedings of the National Academy of Sciences 2017, 114 (40), 10584.

13. Franke, K.; Ross-Messemer, M.; Menck, A.; Hoeller, R. S.; Schmidt, H.; Weilmacht, M., The highly sensitive optical measurement of absolute SAW amplitudes for power flow analysis. IEEE Transactions on Ultrasonics, Ferroelectrics, and Frequency Control 2003, 50 (1), 77-80.

14. Sun, T.; Morgan, H., Single-cell microfluidic impedance cytometry: a review. Microfluidics and Nanofluidics 2010, 8 (4), 423-443. 